Document downloaded from:

http://hdl.handle.net/10251/123280

This paper must be cited as:

Quiles, F.; Belenguer Martínez, A.; Martínez-Zamora, JȦ; Nova-Giménez, V.; Esteban González, H.; Boria Esbert, VE. (2018). Compact Microstrip to Empty Substrate Integrated Coaxial Line Transition. IEEE Microwave and Wireless Components Letters. 28(12):10801082. https://doi.org/10.1109/LMWC.2018.2874280

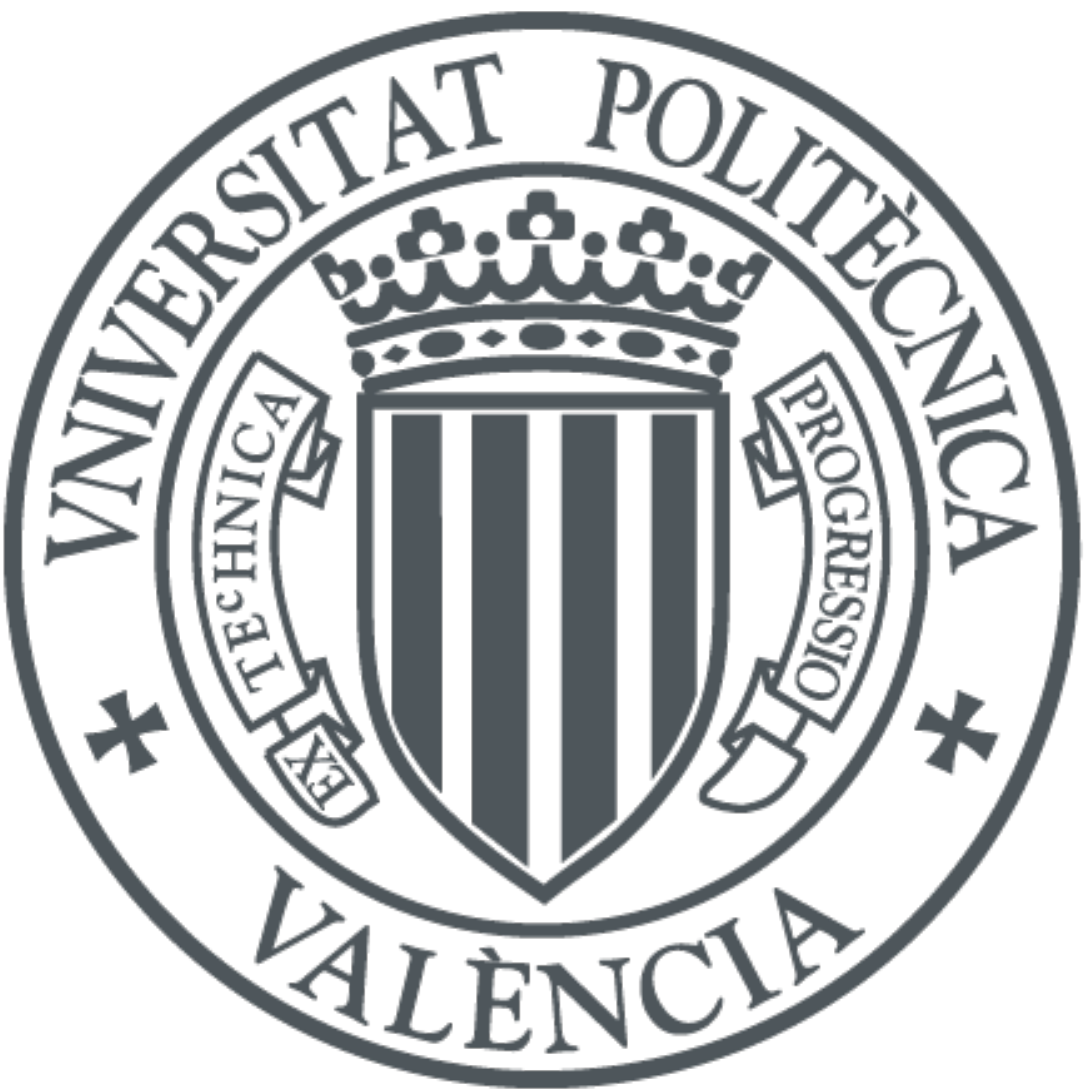

The final publication is available at

http://doi.org/10.1109/LMWC.2018.2874280

Copyright Institute of Electrical and Electronics Engineers

Additional Information 


\title{
Compact Microstrip to Empty Substrate Integrated Coaxial Line Transition
}

\author{
Ferran Quiles $^{1}$, Ángel Belenguer ${ }^{2}$, Juan Ángel Martínez ${ }^{2}$, Vicente Nova ${ }^{1}$, Héctor Esteban ${ }^{1}$, Vicente Boria ${ }^{1}$
}

\begin{abstract}
Substrate integrated waveguides are increasingly being used due to their capability of combining the advantages of planar circuits and traditional waveguides. The development of empty substrate integrated waveguides has substantially reduced the related insertion losses, since waves propagate through air instead of propagating through a lossy dielectric medium. Recently, a new empty coaxial structure, completely built with printed circuit boards and integrated in a substrate, has been proposed. It has been named empty substrate integrated coaxial Line (ESICL). The resulting coaxial line has low cost, easy manufacturing, low radiation, low losses, high-quality factor, and is non dispersive. A transition from grounded coplanar waveguide to ESICL already exists. In this work, a transition from microstrip to ESICL is presented for the first time. In order to demonstrate its feasibility, a back-to-back structure and a bandpass filter have been manufactured and measured.

Index Terms-Substrate integrated waveguide (SIW), empty substrate integrated waveguide (ESIW), transition, bandpass filter, empty substrate-integrated coaxial line (ESICL).
\end{abstract}

\section{INTRODUCTION}

In the quest for developing a new transmission line with the capabilites of the traditional waveguide and the ease of integration of the planar technologies, the first substrate integrated circuit (SIC), the substrate integrated waveguide (SIW), was proposed by Deslandes and Wu [1]. In the SIW, a rectangular waveguide is synthesized in a planar circuit board metallizing via holes that act as the lateral walls of the guide.

Thereafter, a new type of substrate integrated waveguides have appeared, where the dielectric is removed thus significantly decreasing the insertion losses. It is the case of the empty substrate integrated waveguide (ESIW) [2], the air filled substrate integrated waveguide [3], the hollow substrate integrated waveguide (HSIW) [4], or the dielectricless substrate integrated waveguide [5]. All these solutions are one conductor structures that propagate the fundamental $\mathrm{TE}_{10}$ mode, as it happens with the standard rectangular waveguide. Thus, they are dispersive waveguides.

In [6], an empty substrate integrated coaxial line (ESICL) was first presented. With five substrate layers, it creates an empty rectangular coaxial line, which propagates a nondispersive mode with low loss, low radiation, large monomode bandwidth, and mantaining the advantages of the other empty substrate integrated circuits (low cost, low profile, easy manufacturing).

1 Dpto. de Comunicaciones. Universitat Politècnica de València. Camino de Vera, s/n, 46022 Valencia, Spain, Tel.: +34 963877007 (Ext. 88278) e-mail: hesteban@dcom.upv.es

2 Departamento de Ingeniería Eléctrica, Electrónica, Automática y Comunicaciones, Universidad de Castilla-La Mancha, Escuela Politécnica de Cuenca, Campus Universitario, 16071 Cuenca, Spain, e-mail: angel.belenguer@uclm.es
In order to use the ESICL in microwave circuits, it has to be connected to other devices integrated in the same substrate. For that purpose, a transition from grounded coplanar waveguide (GCPW) to ESICL was firstly developed and presented in [7], but no transition from microstrip line has been developed yet. So, in this work a transition from microstrip to ESICL is proposed, and tested with the manufacturing of a back-to-back structure and a bandpass filter.

The paper is organized as follows. Section II presents the structure of the microstrip to ESICL transition and its design procedure. The experimental results of a back-to-back transition and a bandpass filter are presented in Section III. Finally, the main conclusions of the work are discussed in Section IV.

\section{PROPOSED DESIGN}

The new microstrip to ESICL transition presents a simple design and a compact structure, which eases its manufacturing while maintaining a robust device. The proposed structure can be observed in figure 1. The detailed layout of the central layer (layer 2 in figure 1) is presented in figure 2.

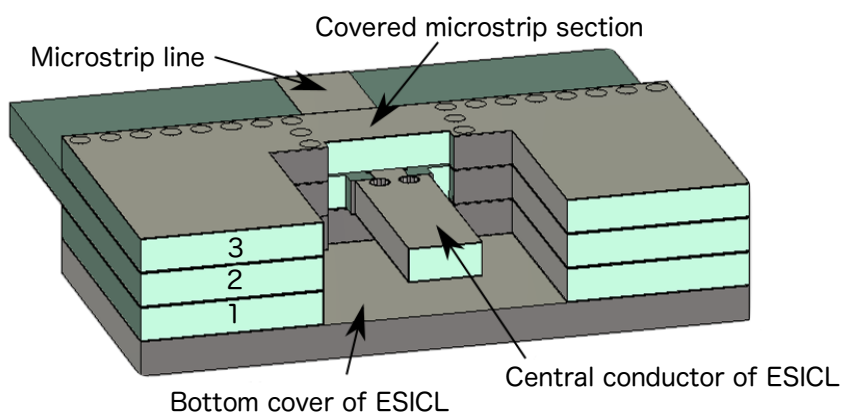

Fig. 1. Structure of the proposed transition from microstrip to ESICL. Layers 1 and 3 separate the central conductor of ESICL from the top and bottom covers. Layer 2 hosts the accessing microstrip line and the central conductor of the ESICL. The top metallic cover of the ESICL has not been depicted.

Starting with a feeding microstrip line, and in order to progressively adapt the fields to the final distribution in the ESICL, the microstrip line is covered in the upper region with a substrate (layer 3 ). Via holes are metallized in layer 3 in this region of covered microstrip line in order to to improve the confinement of the electromagnetic field, and to better adapt the electromagnetic (EM) field distribution to the ESICL. Then, the track is connected to the central conductor of the ESICL through a two-steps aperture (first step of length $l_{c u t}$, total length $l t_{3}$, as shown in the layout of the central layer in figure 2). This last stage is based on [7], which 
has been proved to be an excellent solution for providing a high coupling between both stages. The substrate is narrowed $\left(w_{c u t}\right)$, the bottom metallization is eliminated so that the inner conductor of the ESICL is not connected to the ground, and the microstrip track changes its width to achieve the appropriate impedance. Moreover, two via holes are introduced at the beginning of the central conductor of the ESICL in order to avoid the propagation of electromagnetic fields inside it.

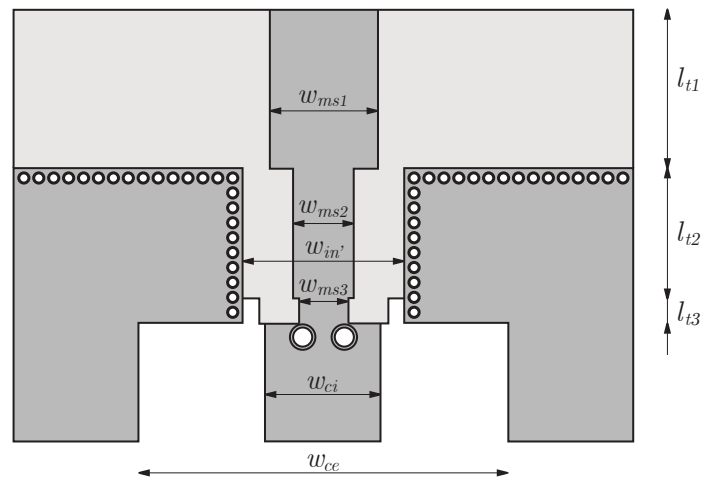

(a)

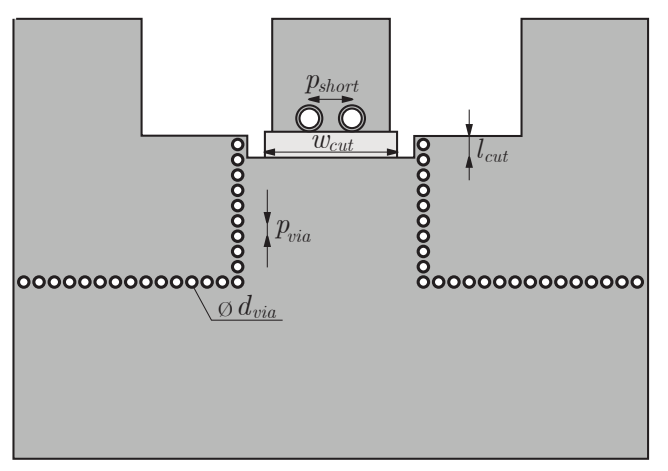

(b)

Fig. 2. Central layer of the microstrip to ESICL transition. (a) Top (b) Bottom.

The proposed design procedure for this transition is as follows. In the first place, the dimensions of the ESICL $\left(w_{c i}\right.$ and $w_{c e}$ ) are chosen to ensure an impedance of $50 \Omega$ (see [8]). Next, the width of the microstrip track in each region $\left(w_{m s_{1}}\right.$, $w_{m s_{2}}$, and $w_{m s_{3}}$ ) is optimized (with a Trust Region algorithm) so that the same characteristic impedance $(50 \Omega)$ is maintained throughout the transition. Then, the lengths of each section $\left(l t_{2}, l t_{3}\right)$ are initially set, respectively, to $\frac{\lambda_{2}\left(f_{0}\right)}{4}$ and $\frac{\lambda_{3}\left(f_{0}\right)}{4}$, that is, to a quarter of the wavelength at the central frequency in each one of the sections. Then, $l t_{2}$, and $l t_{3}$, together with the other design parameters $\left(w_{i n}^{\prime}\right.$, and $\left.l_{c u t}\right)$, are optimized with a Nelder Mead Simplex algorithm, in order to maximize the return losses in the band of interest (in this case from 0 to 20 $\mathrm{GHz}$ ). The parameter $w_{c u t}$ is fixed to $w_{c i}+0,2 \mathrm{~mm}$ in order to ensure ease of manufacturing and mechanical stability.

This design procedure has been applied for two different substrates, the Rogers 4003C substrate (permittivity $\varepsilon_{r}=3.55$, substrate thickness $h=0.813 \mathrm{~mm}$, and copper thickness $t_{s}=$ $17.5 \mu \mathrm{m})$ and the Rogers $5880\left(\varepsilon_{r}=2.20, h=0.508 \mathrm{~mm}\right.$, and $\left.t_{s}=35 \mu \mathrm{m}\right)$. The thickness of the electrolytic metallization layer is experimentally estimated to be $t_{m}=9 \mu \mathrm{m}$.

Table I shows the values of the dimensions of the transition that are fixed, and that are not subject to optimization. $h_{e}$, the height of the ESICL, is fixed by the substrate height and the thickness of the copper layers. Table I also shows the values of the design parameters after optimization. It is worth mentioning that the initial values for some of these parameters $\left(w_{\text {in }}^{\prime}\right.$ and $\left.l_{\text {cut }}\right)$ are extracted from [7].

\begin{tabular}{|c|c|c|}
\hline \multicolumn{3}{|c|}{ Fixed parameters } \\
\hline & $4003 \mathrm{C}$ & 5880 \\
\hline$w c_{i}$ & 1.8171 & 1.1953 \\
\hline$w c_{e}$ & 6 & 4.5 \\
\hline$h$ & 0.813 & 0.508 \\
\hline$t=t_{s}+t_{m}$ & 0.0265 & 0.0265 \\
\hline$h_{e}=3 h+8 t$ & 2.651 & 1.736 \\
\hline$l_{t_{1}}$ & 10 & 10 \\
\hline$w_{m s_{1}}$ & 1.0989 & 1.209 \\
\hline$w_{m s_{2}}$ & 1.0145 & 0.6445 \\
\hline$w_{m s_{3}}$ & 0.8175 & 0.6994 \\
\hline$d_{v i a}$ & 0.5 & 0.5 \\
\hline$p_{v i a}$ & 0.75 & 0.75 \\
\hline$p_{\text {short }}$ & 0.7254 & 0.6 \\
\hline$w_{c u t}=w_{c i}+0.2$ & 2.0171 & 1.3953 \\
\hline \multicolumn{3}{|c|}{ Optimized parameters } \\
\hline & $4003 \mathrm{C}$ & 5880 \\
\hline$l_{t_{2}}$ & 2.0024 & 1.6439 \\
\hline$l_{t_{3}}$ & 0.4769 & 0.5246 \\
\hline$w_{i n}^{\prime}$ & 3.0973 & 3.4589 \\
\hline$l_{\text {cut }}$ & 0.3909 & 0.5 \\
\hline
\end{tabular}

Table I. Values of the fixed and design parameters (in mm) of the transition.

The length of the transition $\left(l_{t_{2}}+l_{t_{3}}\right)$ is, respectively, $3.64 \mathrm{~mm}$ and $2.17 \mathrm{~mm}$, for the Rogers $4003 \mathrm{C}$ and the Rogers 5880 designs. These lengths are $0.12 \lambda$ and $0.24 \lambda$ at $10 \mathrm{GHz}$.

\section{RESULTS}

The simulated performance of the two transition designed in the previous sections with the Rogers $4003 \mathrm{C}$ and 5880 substrates can be seen in figure 3 . The values of table I have been used. It can be observed that return losses are greater than $30 \mathrm{~dB}$ in the whole design band for both designs, which was the optimization goal, and that the insertion losses are not greater than $0.45 \mathrm{~dB}$ for the $4003 \mathrm{C}$ substrate, and not greater than $0.2 \mathrm{~dB}$ for the thinner 5880 substrate, where the microstrip line is less radiative.

A back-to-back structure of the transition with Rogers 4003C has been manufactured, as shown in figure 4. The measurements are compared with simulations in figure 5, where a good agreement can be observed.

In addition to the back-to-back transition prototype, a coupled cavities bandpass filter has been designed and manufactured in order to test the performance of the proposed transition in a practical application. The manufactured filter is shown in figure 6 , and the comparison of simulation and measurements are depicted in figure 7 . More than $15 \mathrm{~dB}$ of return losses and less than $1 \mathrm{~dB}$ of insertion losses in the passband (with center frequency and bandwidth of $15 \mathrm{GHz}$ and 10 $\mathrm{GHz}$ ) is obtained in the measured response. 


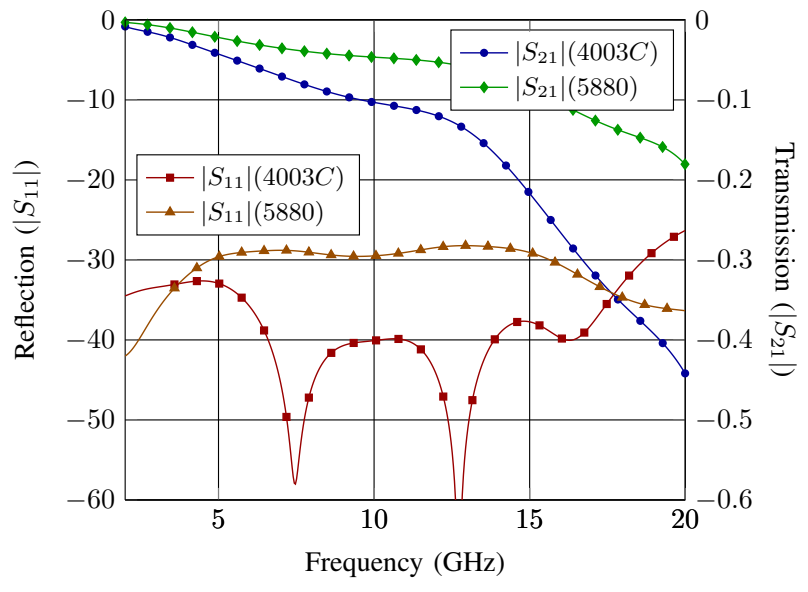

Fig. 3. Simulated scattering parameters of the transition with Rogers $4003 \mathrm{C}$ and 5880 substrates.

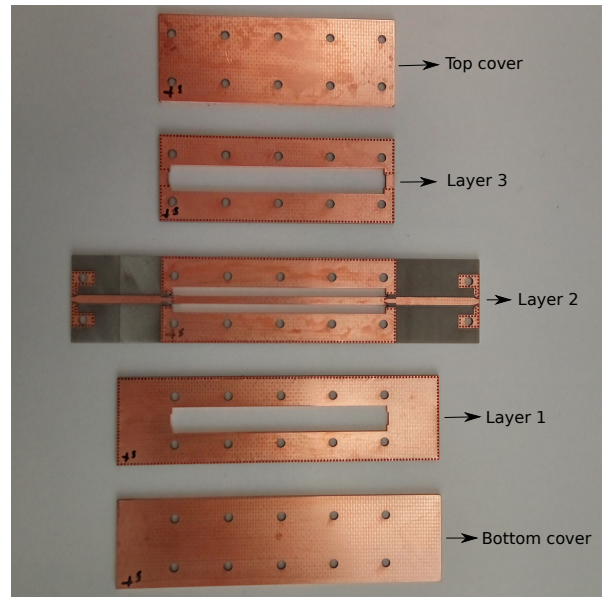

Fig. 4. Back-to-back manufactured prototype.

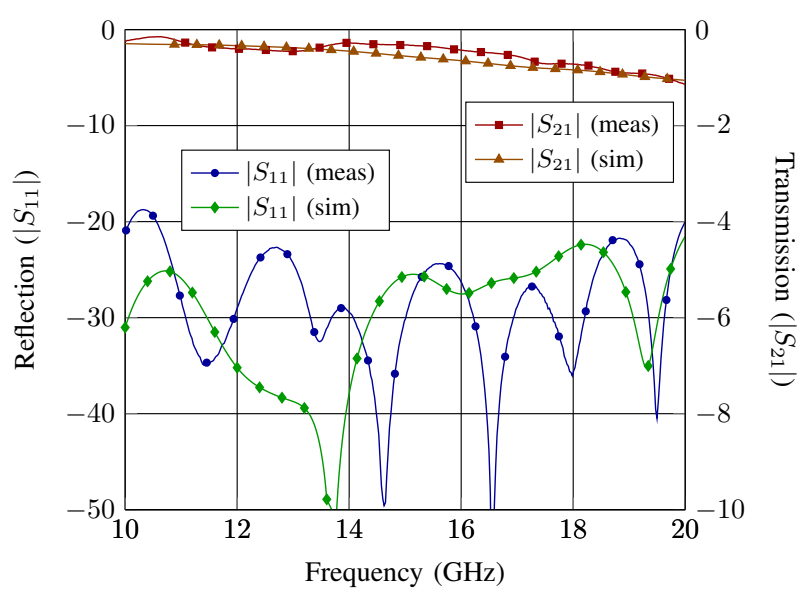

Fig. 5. Comparison of measurements and simulations of the the back-to-back transition prototype.

\section{CONCLUSIONS}

A transition from microstrip to ESICL has been proposed for the first time, based on a simple and compact solution. Measured return losses greater than $18 \mathrm{~dB}$, and insertion

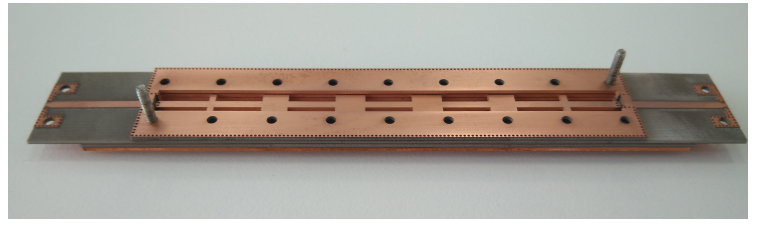

Fig. 6. Bandpass ESICL filter.

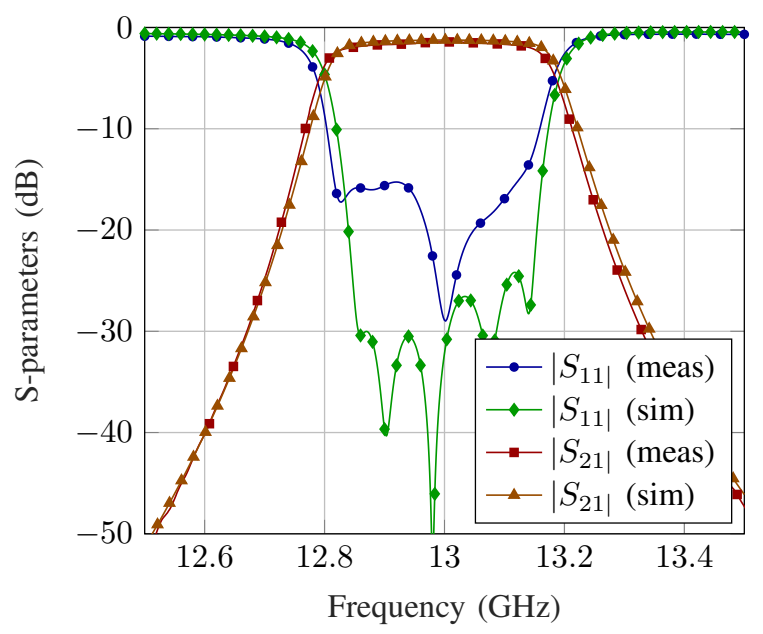

Fig. 7. Results of the bandpass ESICL filter.

losses lower than $1 \mathrm{~dB}$ have been obtained in all the usable frequency band with the back-to-back manufactured prototype. The transition has also been successfully tested for connecting an ESICL filter to accessing microstrip lines.

\section{REFERENCES}

[1] D. Deslandes and K. Wu, "Integrated microstrip and rectangular waveguide in planar form," Microwave and Wireless Components Letters, IEEE, vol. 11, no. 2, pp. 68-70, Feb 2001.

[2] A. Belenguer, H. Esteban, and V. Boria, "Novel empty substrate integrated waveguide for high-performance microwave integrated circuits," Microwave Theory and Techniques, IEEE Transactions on, vol. 62, no. 4, pp. 832-839, April 2014.

[3] F. Parment, A. Ghiotto, T.-P. Vuong, J.-M. Duchamp, and K. Wu, "Air-filled substrate integrated waveguide for low-loss and high powerhandling millimeter-wave substrate integrated circuits," Microwave Theory and Techniques, IEEE Transactions on, vol. 63, no. 4, pp. 1228-1238, April 2015.

[4] L. Jin, R. Lee, and I. Robertson, "Analysis and design of a novel low-loss hollow substrate integrated waveguide," Microwave Theory and Techniques, IEEE Transactions on, vol. 62, no. 8, pp. 1616-1624, Aug 2014.

[5] F. Bigelli, D. Mencarelli, M. Farina, G. Venanzoni, P. Scalmati, C. Renghini, and A. Morini, "Design and fabrication of a dielectricless substrate-integrated waveguide," Components, Packaging and Manufacturing Technology, IEEE Transactions on, vol. 6, no. 2, pp. 256-261, Feb 2016.

[6] N. Jastram and D. Filipovic, "PCB-based prototyping of 3-D micromachined RF subsystems," Antennas and Propagation, IEEE Transactions on, vol. 62, no. 1, pp. 420-429, Jan 2014.

[7] A. Belenguer, A. Borja, H. Esteban, and V. Boria, "High-performance coplanar waveguide to empty substrate integrated coaxial line transition," Microwave Theory and Techniques, IEEE Transactions on, vol. 63, no. 12, pp. 4027-4034, Dec 2015.

[8] T. S. Chen, "Determination of the capacitance, inductance, and characteristic impedance of rectangular lines," IRE Transactions on Microwave Theory and Techniques, vol. 8, no. 5, pp. 510-519, September 1960. 